\title{
Biomimetic Synthesized Conductive Copolymer EDOT-Pyrrole Electrodes for Electrocardiogram Recording in Humans
}

\author{
Manuel Eduardo Martínez-Cartagena ${ }^{1 *}$, Juan Bernal-Martínez², Carlos Alberto Aranda-Sánchez², \\ Arnulfo Banda-Villanueva ${ }^{3}$, José Luis Gonzalez-Zapata ${ }^{3}$, Antonio Ledezma-Pérez ${ }^{1}$, \\ Alfredo Aguilar-Elguezabal ${ }^{4}$, Jorge Romero-García ${ }^{1 \#}$ \\ ${ }^{1}$ Advanced Materials Department, Research Center in Applied Chemistry (CIQA), Saltillo, Coahula, México \\ ${ }^{2}$ Laboratory in Biomedicine and Nanotechnology, Aguascalientes, Aguascalientes, México \\ ${ }^{3}$ Department of Reactions Engineering, Research Center in Applied Chemistry (CIQA), Saltillo, Coahula, México \\ ${ }^{4}$ Department of Engineering and Chemistry of Materials, Research Center in Advanced Materials (CIMAV), Chihuahua, México \\ Email: *eduardocartaa@gmail.com
}

How to cite this paper: Martínez-Cartagena, M.E., Bernal-Martínez, J., Aranda-Sánchez, C.A., Banda-Villanueva, A., Gonzalez-Zapata, J.L., Ledezma-Pérez, A., Aguilar-Elguezabal, A. and Romero-García, J. (2021) Biomimetic Synthesized Conductive Copolymer EDOTPyrrole Electrodes for Electrocardiogram Recording in Humans. Journal of Materials Science and Chemical Engineering, 9, 19-40. https://doi.org/10.4236/msce.2021.910003

Received: September 18, 2021

Accepted: October 26, 2021

Published: October 29, 2021

Copyright () 2021 by author(s) and Scientific Research Publishing Inc. This work is licensed under the Creative Commons Attribution International License (CC BY 4.0).

http://creativecommons.org/licenses/by/4.0/

\begin{abstract}
We report on electrodes fabricated with EDOT-Pyrrole copolymer through electrophoretic deposition and used for recording and sensing bio-electrical signals. We measured the electrical properties of the copolymer deposited on a stainless-steel substrate, and we performed Cyclic Voltammetry (CV) and Scanning Electron Microscopy (SEM) studies to characterize the morphological properties and copolymer distribution on the metal surface. We found that electrodes fabricated with EDOT-Pyrrole copolymer exhibit a high signal-to-noise ratio as well as an accurate and stable conductivity compared with other commonly used electroconductive polymers. Stainless-steel-coated EDOT-Pyrrole electrodes are suitable to record electrocardiograms in humans with high resolution comparable to standard silver-electrodes.
\end{abstract}

\section{Keywords}

Electrocardiogram, Bioelectrical Signals, Biomimetic, Conductive Polymers

\section{Introduction}

Since Einthoven's seminal work on recording the first modern electrocardiogram, the development and improvement of the technique have been continuous and sustained. The electrocardiograph is one of the most useful and invaluable 
clinical tools which provides physicians the preliminary diagnosis of cardiac complaints. The electrodes are a critical part of the electrocardiogram recording (ECG) and their evolution in the past century has been dramatic, from cylindrical electrolyte electrodes to the wearable electrodes commonly used in the last few years. Typically, an ECG electrode is made of silver/silver chloride (Ag/ $\mathrm{AgCl}$ ), due to low impedance, high conductivity, and electrical stability in its system. However, the shape and size of this kind of electrode are limited due to biocompatibility effects.

To overcome the biocompatibility effects, recent advances in electrode materials have been made, in order to achieve comfortable, flexible and biocompatible electrodes. A wide variety of materials have been employed, such as carbon nanomaterials [1], carbon nanotubes [2], metallic nanoparticles [3], inorganic semiconductors [4], and electroconductive polymers. Conductive polymers are very promising for electrode materials because they are soft, electrically conductive, low cost, easily synthesized, and biocompatible with human tissues [5] [6]. The most used conductive polymer material is poly (3,4-ethylenedioxythiophene): polystyrene sulfonate (PEDOT:PSS). Several scientific publications have indicated the accuracy and effectiveness of PEDOT:PSS as ECG electrode material. Bihar et al. reported on PEDOT:PSS electrodes inkjet-printed on paper for ECG recording [7]. Rossetie et al. coated electrochemical stainless steel microwires with PEDOT:PSS for electromyogram recording in rats [8]. Zahed et al. manufactured PEDOT:PSS loaded laser-induced graphene (LIG) electrodes for ECG recording [9]. Sihna et al. reported that screen-printed PEDOT:PSS textile electrodes for ECG under dry conditions have similar recording parameters to commercial $\mathrm{Ag} / \mathrm{AgCl}$ electrodes [10]. Mihara et al. fabricated PEDOT:PSS/poly (styrene-butadiene-styrene) (SBS) waterproof bilayer nanosheet films for a comfortable waterproof bioelectrode [11]. Lee et al. developed biocompatible, selfadhesive hydrogel electrodes for ECG measurements prepared from gelatin crosslinked with genipin, as a natural water-soluble crosslinking agent with less toxicity [12]. Trindade et al. fabricated in situ PEDOT textile electrodes for continuous monitoring ECG [13].

One of the most fundamental characteristics of bioelectrodes is their biocompatibility. PEDOT:PSS, as well as other conductive polymers that are usually biocompatible. However, it has been found that the polymer synthesis method may affect the biocompatibility of the material as the common chemical oxidative polymerization pathway produces a broad of toxic residues that are difficult to remove after synthesis. An alternative is an electrochemical synthesis, however through this technique the size and shape of the fabricated electrodes is limited, and it is not possible to generate high mass loading. In the last few years, a new synthesis approach emerged based on the enzymatic activity for catalysis of non-biological reactions like the conductive polymer synthesis [14]. This method produces polymers with much fewer toxic residues. However, the high susceptibility of the enzymes under the typical low $\mathrm{pH}$ conditions demanded by 
conductive polymer synthesis reduces the success of the approach. Biomimetic synthesis overcomes enzymatic synthesis limitations. It has been demonstrated that biomimetic synthesis allows to mimicry enzyme catalyst function using organic molecules to bypass the enzyme's fragility, which results in a robust pathway for conductive polymer synthesis compared to enzymatic synthesis [15]. The Hematin molecule has been used by our research team for the biomimetic synthesis of polyaniline and poly-pyrrole (PPY) [15]. The second major problem concerning conductive polymer bioelectrodes is the manufacturing method. Several approaches have been proposed in the last 30 years, including, spincoating, inkjet printing, electrochemical deposition, 3D printing, in situ chemical synthesis, screen printing, photolithography, radiation synthesis, etc [10] [16]-[22]. Here we propose the use of electrophoretic deposition method for manufacturing conductive polymer electrodes. In this work, we report for the first time, ECG recordings made with electrodes of biomimetically synthetized 3,4-ethylenedioxythiophene-Pyrrole (EDOT-PY) copolymer electrophoretically deposited onto stainless steel sheets. The SNR is close to commercial $\mathrm{Ag} / \mathrm{AgCl}$ electrodes, with lower skin-impedance than $\mathrm{Ag} / \mathrm{AgCl}$ electrodes.

\section{Materials and Methods}

\subsection{Biomimetic Synthesis of Homopolymers and Copolymers Doped with PSS}

In our experiments, $1 \mathrm{~mL}$ of a Dimethyl sulfoxide (DMSO)-Hematin solution $(100 \mathrm{mg} / \mathrm{mL})$ and $200 \mathrm{mg}$ of $\operatorname{EDOT}(3,4$-ethylenedioxythiophene) were added to $20 \mathrm{~mL}$ of 1:40 PSS/TSA solution $\mathrm{pH} 2$ (70,000 g/mol PSS obtained by RAFT). The emulsion was stabilized for 2 hours under magnetic stirring, then pyrrole (Py) (0.1 molar fraction, to adjust the molar fraction of monomers as EDOT 0.9 - Py 0.1) as added and constant stirring was maintained at $1000 \mathrm{rpm}$ for 2 hours at room temperature. Polymerization begins by microdosing $200 \mu \mathrm{L}$ of $30 \%$ $\mathrm{H}_{2} \mathrm{O}_{2}$ for 1 minute, keeping the system at $0^{\circ} \mathrm{C}$. The temperature and stirring conditions $\left(0^{\circ} \mathrm{C}, 250 \mathrm{rpm}\right)$ were sustained for 18 hours. Each copolymer was frozen at $-25^{\circ} \mathrm{C}$ and lyophilized for 24 hours, then resuspended in cold acetone, filtered on a $0.25 \mu \mathrm{m}$ PTFE membrane, and multiple acetone washes were running over the product until a colorless filtered liquid was obtained. Finally, the product was dried at $70^{\circ} \mathrm{C}$ for 12 hours. The synthesis of the homopolymers and copolymers without PSS, doped only with p-Toluenesulfonic acid (TSA), was carried out keeping the same synthesis conditions described above.

\subsection{Electrode Manufacturing: Electrophoretic Deposit}

The biomimetically synthesized conjugated copolymer (CCBS) chosen for electrophoretic deposition was EDOT-PY in a molar fraction $0.9-0.1$, as well as their respective homopolymers, all doped with PSS according to the synthesis methodology described above. Colloidal dispersions were prepared at a concentration of $10 \mathrm{mg} / \mathrm{mL}$ using a $500 \mathrm{mM}$ PBS (phosphate buffer solution $\mathrm{pH}$ 7) 
aqueous solution as a medium dispersant and sonicated for 15 minutes. The deposit was carried out using a glass container at a capacity of $90 \%(25 \mathrm{~mL}$ of CCBS dispersion), with a surgical grade stainless-steel plate work electrode (0.1 $\mathrm{mm}$ thick, $0.75 \mathrm{~cm}^{2}$ area) separated by $1 \mathrm{~cm}$ from a Pt wire coil as a counter electrode $(4 \mathrm{~cm})$. Before each experiment, the electrodes were washed by sonication in isopropanol for 30 seconds, then dried with compressed air. The electrophoretic deposit (EPD) was performed at $12 \mathrm{~V}$ (constant voltage source PowerPac Biorad) at room temperature with magnetic stirring (100 rpm). Different deposit times (5 - $120 \mathrm{~min}$ ) were evaluated. After each deposit, each electrode was dried with compressed air and stored in hermetic packaging. The amount of mass deposited on the stainless-steel electrode was recorded for the different deposit times of the EPD method described above. At different intervals, the electrode was weighed using an analytical balance (SE2ultra-microbalance Sartorius with a resolution of $0.1 \mathrm{mg}$ ). The electrical resistance presented by the deposit at each interval was measured using a Caitlyn electrometer.

\subsection{Cyclic Voltammetry Studies}

The cyclic voltametric (CV) measurements were carried out using a potentiostat (Rodeostat, IO Rodeo Smart Lab Technology) and a three-electrode cell where the working electrode (WE) corresponds to an electrode manufactured by any of the mentioned methods or a tablet of $7 \mathrm{~mm}$ diameter bulk polymer. The counter electrode (CE) consists of a platinum wire and $\mathrm{Ag} / \mathrm{AgCl}$ was used as a reference electrode (RE). The electrolytes used were $0.5 \mathrm{M} \mathrm{NaCl}$ and $0.5 \mathrm{M}$ PBS. Once the electrodes were submerged in the electrolyte, $\mathrm{N}_{2}$ was bubbled in for 20 minutes. The operating conditions consisted of a potential window of $-0.7 \mathrm{~V}$ to $0.7 \mathrm{~V}$, and sweep speeds of $100 \mathrm{mV} / \mathrm{s}$ for 10 cycles.

\subsection{Specific Capacitance}

The specific capacitance was determined by cyclovoltammetry with the configuration cell and the electrolyte mentioned above. A potential window was set at $-0.7 \mathrm{~V}$ to $0.7 \mathrm{~V}$ and a sweep speed of $100 \mathrm{mV} / \mathrm{s}$, which was calculated according to the equation

$$
C s=S / 2 \Delta U V
$$

where $C s$ is the specific capacitance, $S$ is the integrated area of the voltametric curve (expressed as $\mathrm{mA} \cdot \mathrm{V} / \mathrm{cm}^{2}$ ), $\Delta U$ is the potential window used, and $V$ is the sweep speed (expressed as $\mathrm{V} / \mathrm{s}$ ).

\subsection{Scanning Electron Microscopy and Elemental Analysis}

Scanning electron microscopy (SEM) images and elemental analysis (EDX) mapping were obtained using a LEO (Zeiss) 1540XB FIB scanning electron microscope with a gun emission field of 3 to $15 \mathrm{kV}$ and a $5 \mathrm{~mm}$ working distance from the sample holder to the optical system. The EDX microanalysis was obtained using an Oxford Inca Energy 350X-MAX 50, with linear resolution of 127 
$\mathrm{eV}$ in $\mathrm{Mn} \mathrm{Ka}$ from 1 to 100,000 cps., The detection and quantification of elements are from atomic number 4 (Beryllium).

\subsection{Transmission Electron Microscopy}

The transmission electron microscopy (TEM) images were taken on a Titan Fei Thermofischer instrument with an acceleration voltage of $200 \mathrm{kV}$. The samples were prepared by immersing lacey-carbon grids in an isopropanol suspension, allowing them to dry in a vacuum oven for 2 hours at $60^{\circ} \mathrm{C}$.

\subsection{Infrared Spectroscopy}

Fourier transform infrared (FTIR) spectra were acquired using a Thermo Fischer Scientific FTIR Spectrophotometer in the attenuated total reflectance (ATR) mode using a diamond crystal. The sample does not require preparation. The powder is placed on the surface of the glass and the measurement is carried out. The spectra were acquired taking an average of 32 scans with a resolution of 4 $\mathrm{cm}^{-1}$ within a range between $400 \mathrm{~cm}^{-1}$ to $4000 \mathrm{~cm}^{-1}$.

\subsection{Powder X-Ray Diffraction}

The powder $\mathrm{x}$-ray diffraction (PXRD) patterns were acquired using a Brucker D8 Advance Diffractometer with a $\mathrm{Cu}$ Ka radiation source $(\lambda=1.5418 \AA$ ). The powdered samples were placed in a standard sample holder. Measurements were made at intervals of $0.02^{\circ}$ at a scanning speed of $10^{\circ} / \mathrm{min}$ from $2 \theta=2^{\circ}$ to $82^{\circ}$.

\subsection{Electrophysiological Assays}

The ECG was recorded with a MP36R Biopac unit and an RHD2000 electrode adapter plate. Electrophysiological ECG recordings were performed using an EDOT-PY 0.9 - 0.1:PSS/PO ${ }_{4}$ EPD electrode, and a Biopac electrophysiological commercial-grade $\mathrm{Ag} / \mathrm{AgCl}$ electrode was used as a control. The tests were carried out with a 3-electrode configuration: the reference electrode (RE) was placed on the right ankle, the working electrode (WE) and counter electrode (CE) were placed on the left and right arm respectively, $5 \mathrm{~cm}$ from the wrists. Each test used a trio of equal electrodes (flat stainless steel $1.5 \times 0.5 \mathrm{~cm}$ electrodes). An electrophysiological gel drop was added to each electrode before starting the test. The test subject (female, average weight) remained seated and at rest while the signal was allowed to stabilize for 15 minutes, and any type of movements was avoided to not add breathing or shaking artifacts.

\subsection{Signal-to-Noise Ratio Analysis}

The value of the signal-to-noise ratio (SNR) was extracted from the electrophysiological ECG recordings. A MATLAB function is applied to the data to obtain the root mean square (RMS) value of the signal of interest divided by RMS value of electronic noise according to the following formula: 


$$
\mathrm{SNR}=10 \log \frac{\sqrt{(\text { RMS }: \text { signal })^{2}}}{\sqrt{(\text { RMS }: \text { noise })^{2}}}
$$

The thermal noise voltage was calculated with the equation:

$$
\mathrm{Vn}=\sqrt{4 \mathrm{kTBR}}
$$

where $\mathrm{k}$ is the Boltzmann's constant in $\mathrm{J} / \mathrm{K}$, $\mathrm{T}$ is the temperature in degrees $\mathrm{K}, \mathrm{B}$ is the noise bandwidth value, and $\mathrm{R}$ is the impedance in Ohms.

\section{Results and Discussion}

\subsection{Polymer Composition through Fourier Transform Infrared Spectrometry}

Table S1 (supporting information) presents the main bands identified in the different polymers analyzed. When analyzing PEDOT-Hematin, the appearance of a band related to the asymmetric stretching $\mathrm{C}=\mathrm{C}$ of the ring [23] is observed at $1539 \mathrm{~cm}^{-1}$. Likewise, some signals associated with the symmetric stretching of the ring were also not observed, which could mean a decrease in conjugation in the chain related to defects such as branching and crosslinking, which in general terms affects the conductivity of the polymer. In general, when comparing the spectra of PEDOT or Polypyrrole (PPY) (Figure 1), most of the bands have remarkable similarity regardless of the chemical skeletons of PPY and PEDOT, which indicate that the hematin does not exert a significant effect on the chemical structure of the homopolymers of PEDOT and PPY just as a catalyst would. Figure 1 shows the Fourier transform infrared (FTIR) spectra of the EDOT-PY copolymers: $0.9-0.1,0.7-0.3,0.5-0.5$ and $0.3-0.7$ biomimetically synthesized in the presence of Hematin. In the case of the PEDOT PSS spectrum, the existing bands corroborate the chemical structure of the polymer reported in the literature. Contributions from PSS are also observed, specifically from the $-\mathrm{SOx}$ group, which indicates that the doping process with PSS was successful. The spectrum of PPY:PSS shows the characteristic bands observed for PPY doped with TSA as well as how groups of the polymer were observed. When analyzing the bands of the EDOT-PY:PSS copolymer (Table S1), the formation of the copolymer is corroborated, with respect to the molar composition. Similarities can be observed in practically identical positions, which confirm the chemical structure of the synthesized copolymer and reproduces what was observed in the case of doping with TSA. This can be attributed to the increase in doping ions. According to Sandoval et al., the change in the structure of the doping causes small variations in intensity and position of some bands, especially at frequencies in the region between $1500-1400 \mathrm{~cm}^{-1}$. This coincides with our experimental observations, and some may even disappear, specifically those related to neutral segments of the chain. The variations registered in the FTIR spectrum may indirectly be an indication of the increase of doping in the synthesized structure as well as in the presence of polyanions and an organic acid just as it is with TSA. One interesting characteristic of the spectra is that regardless of the dopant type, 

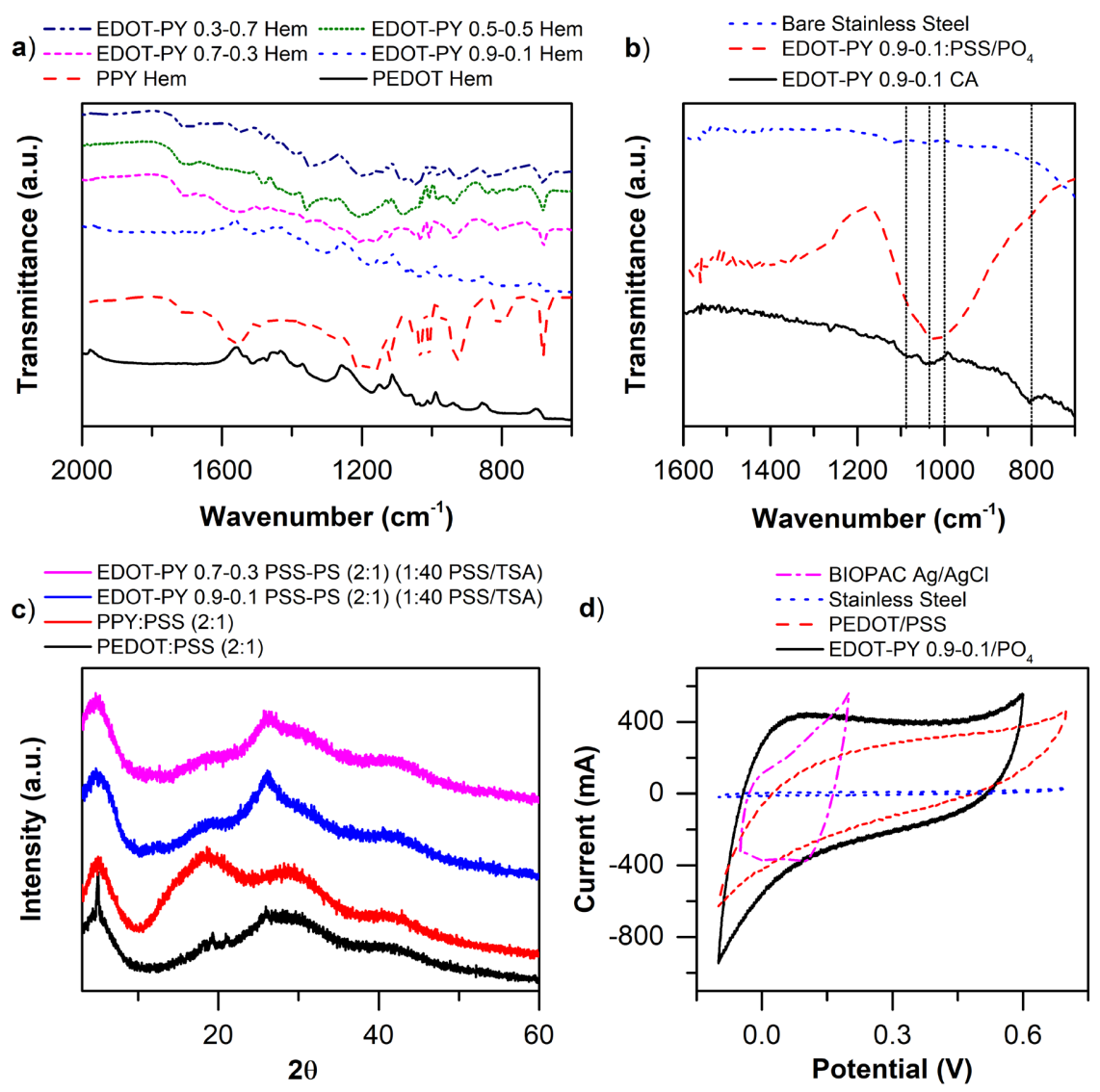

Figure 1. (a) FTIR-ATR spectra of PEDOT:PSS, PPY:PSS and EDOT-PY:PSS copolymers (CCBS) biomimetically catalyzed in the presence of Hematin. (b) FTIR-ATR spectrum of EDOT-PY 0.9 - 0.1 electrodes generated by metal surface coating assisted by electrophoretic deposition (c) X-ray powder diffractograms obtained for PEDOT:PSS, PPY:PSS and EDOT-PY:PSS copolymers biomimetically synthetized. (d) Cyclic voltammograms obtained for PEDOT:PSS and EDOT-PY 0.9 - 0.1:PSS electrophoretically deposited on stainless steel.

the region between 1200 and $1000 \mathrm{~cm}^{-1}$ is equivalent to the different products. This is not a strange result since it would be expected that a structure with a certain chemical character is not dependent on the dopant to retain similar chemical functionalities. Thus, it confirms that the EDOT-PY copolymers showed their own FTIR spectral fingerprint under the conditions of the synthesis investigated.

It is worth mentioning that depending on the molar relationship, there are positional variations in the region between 1500 and $1400 \mathrm{~cm}^{-1}$. This could mean changes in the doping of the chain and in the conjugation of the skeleton, since at a lower content of PEDOT, the bands related to $\mathrm{C}=\mathrm{C}$ the stretching of the ring change, and in the case of the copolymer $0.5-0.5$, they decrease even more remarkably, so much that they cannot be assigned with precision. On the other hand, in the region that comprises $1300-1000 \mathrm{~cm}^{-1}$, there is a high correlation of the $S$ vibrational modes of the present functionalities that indicate equivalence at the structural level. 
In the case of simultaneous doping with TSA and PSS, it is determined that they present comparable spectral patterns. This result illustrates the direct effect that co-doping has. The explanation is likely that a considerable increase in p-type doping of the structure has the consequence of homogenizing the oxidation state of the chain and gives rise to practically identical vibrational modes, because they result from equivalent molecular segments. However, a slight decrease in the intensities of the bands between $1500-1400 \mathrm{~cm}$ can be observed in the $0.5-0.5$ copolymer. This is not as drastic as the case of single doping with PSS, and such an understanding could be relevant for demonstrating the change in the oxidation state of the polymer through the previously mentioned variation in the bands, and which has been suggested previously by Sandoval et al.

Table S1 summarizes the bands identified for each copolymer. Copolymer 0.9 0.1 shows an excellent coincidence with some bands obtained for homopolymers, which means that there are contributions from both chemical structures to the polymeric skeleton, consistent with what was reported by Bruno et al. [24] In elemental terms, this suggests the successful formation of the copolymer from the chemical point of view where the catalyst does not exert a significant effect. The molar ratios (0.7 - 0.3, $0.5-0.5$, and 0.3 - 0.7) show bands of both PEDOT and PPY that confirm the presence of the copolymers. Thus, FTIR spectroscopy allows us to conclude that neither the catalyst nor the type of dopant exerts a significant change in the chemical structure of the copolymer EDOT-PY, which is only modified when the monomer molar ratio changes.

Figure 1(b) presents the deposition of the biomimetically synthesized copolymer EDOT-PY 0.9 - 0.1 and 0.7 - 0.3 carried out on stainless steel surfaces assisted by electrophoresis. The coatings exhibit intense bands at 72:1000 and 1130 $\mathrm{cm}^{-1}$ associated with stretching and deformation of $\mathrm{SOR}$ and $\mathrm{SO}_{2}$ bonds at 1250 $\mathrm{cm}^{-1}$ with $\mathrm{CN}$ bonds. The bands between 1330 and $1450 \mathrm{~cm}^{-1}$ are associated with stretching and deformation of the $\mathrm{CC} \mathrm{sp}{ }^{3}$ and $\mathrm{sp}^{2}$ of the heterocycle, and at 1640 $\mathrm{cm}^{-1}$ the presence of carbonyl groups. It is reasonable to conclude that the methods work for coating stainless steel because the EDOT-PY PSS/PO $\mathrm{P}_{4}$ was deposited cathodically and not anodically as would be expected. Also, the coating shows a wide and intense band centered at $1023 \mathrm{~cm}^{-1}$, which could be associated with the co-deposition of phosphate on the surface [25], which masks or overlaps the vibrational contributions of the copolymer.

Figure 1(c) shows the PEDOT, PPY and EDOT-PY diffractograms. In the case of PEDOT-PSS (2:1) the diffractions become wider because the PSS, being a semicrystalline polymer, introduces a higher level of amorphousness to the structure. Despite this, extremely intense and narrow diffraction maxima can be observed at $2 \theta=4.9^{\circ}$, which could indicate a significant increase in the order between adjacent segments on the XY plane [26]. In the case of PPY:PSS (2:1) the diffractions are extremely wide. However, diffraction at approximately $2 \theta=$ $30^{\circ}$ does not appear when doped only with TSA, so it could be associated with chain stacking of PSS [26] [27] [28] [29]. The homopolymer and copolymer diffractograms show four widened diffractions, which indicate an increase in the 
disorder of the structure due to both, the loss of planarity introduced by the copolymer, and the presence of PSS [30]. In the case of the polyanion PSS and TSA, an interesting effect occurs in the plane associated with chain stacking $(2 \theta=$ $\left.26.2^{\circ}\right)$, since it narrows the diffraction maxima, which indicates higher order in the stacking of chains, and this could possibly increase the electrical conductivity of the material. This is likely either due to the shape and size of the polyanion nanoparticles that can promote a higher order, or that serves as nucleation and growth points for crystalline nanodomains [31]. In general, CCBS diffractograms present four wide signals centered on: $2 \theta=5,16.7,28.6$ and $41.1^{\circ}$ [32] [33] [34]. These diffractions are very broad and rounded, which indicate that the PSS introduces a high degree of amorphousness to the structure. However, the $2 \theta=28.6^{\circ}$ diffraction maxima is sharper compared with the homopolymers probably because the structure of the copolymer generates well defined crystalline stacking microdomains.

\subsection{Cyclic Voltammetry of Different Electroconductive Materials and Electrical Properties of Electroconductive Copolymers}

Figure 1(d) shows the cyclic voltammograms obtained for PEDOT:PSS and EDOT-PY 0.9 - 0.1:PSS electrophoretically deposited on stainless-steel using a 50 mM PBS electrolyte. In orderto check, if it was functional to generate electrophoretic coatings under conditions of very low toxicity, we proposed the use of PBS to favor electrophoretic deposition. This is due to reports in the literature on the use of organic and inorganic phosphates [35] that act as fixatives in the electrophoretic deposit of colloids. The PEDOT:PSS electrophoretic deposited voltammogram (Figure 1(d)) exhibits a similar shape compared with PEDOT:PSS in bulk. The use of the PBS electrolyte probably promotes in situ $\mathrm{FePO}_{4}$ electrosynthesis that helps to entrap the polymer cathodically. There is a tendency for copolymer mobilization to the cathode even when the particles have a negative $\mathrm{Z}$ potential. One hypothesis for this is due to adsorption and ionic exchange phenomena induced by protons, acid phosphate ions and $\mathrm{Fe}^{2+} \mathrm{Ca}-$ tions on the surface of the polymer; nanoparticles modify the surface potential [36] and transport the polymer to the cathode surface, generating a coalescence and layer growth.

Figure 2(a) shows the electrophoretic deposition kinetics of EDOT-PY 0.9 0.1 :PSS on stainless-steel electrode. In the presence of PBS, a quasi-linear increase in the mass can be observed over time, reaching up to $8 \mathrm{mg} / \mathrm{cm}^{2}$ after 60 minutes. However, an increase in the resistance of the electrode with an increase in the mass deposit was observed, therefore deposit times of less than 60 minutes are desirable, ensuring the maximum amount of deposited mass with the least possible electrical resistivity. The EDOT-PY 0.9 - 0.1: $\mathrm{PSS}_{\mathrm{PO}_{4}}$ voltammogram (Figure 1(d)) has a typical quasi-rectangular shape of highly capacitive surfaces with low resistivity, or in other words, non-faradaic (ECDL) plus faradaic process kinetics that contributes to the net's electrochemical response of the material [37]. When compared to the voltametric curve of the uncoated 

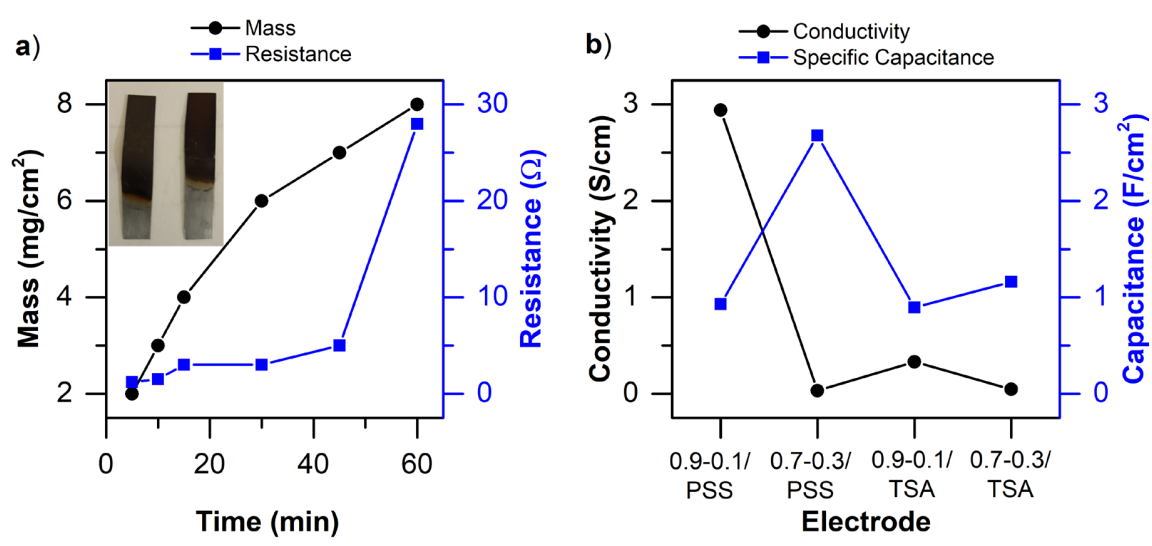

Figure 2. (a) Electrophoretic deposition kinetics of EDOT-PY 0.9 - 0.1:PSS on the stainless-steel electrode. (b) Electrical conductivity copolymers doped with PSS or TSA, and the respective specific capacitance obtained.

stainless-steel electrode (blue curve, Figure 1(d)) with the voltammogram of the copolymer deposit, the minimum capacitance contributed by the metallic surface of the steel is evident. Therefore, the majority of the additional surface capacitance is attributed to the electrophoretically deposited copolymer [38]. The data above shows that the electrophoretic deposition of the EDOT-PY copolymer with PBS as an electrolyte and fixative was successful. The voltammogram obtained from a conventional $\mathrm{Ag} / \mathrm{AgCl}$ electrode exhibits a capacitive behavior (ECDL), which is consistent with a high surface area and consequently a satisfactory signal-to-noise ratio (SNR). However, the voltametric evaluation shows that its potential window is very small with the PBS electrolyte $(-0.045$ to $0.2 \mathrm{~V}$ vs. $\mathrm{Ag} / \mathrm{AgCl})$, which is detrimental, since $\mathrm{Ag} / \mathrm{AgCl}$ electrodes have been shown to release reactive oxygen species [39] [40] when used as a stimulation electrode in cells or microorganisms due to a narrow zone of electrochemical stability [41]. Polymer-coated electrodes should not present such problems since their operating window is wide ( -0.7 to $0.7 \mathrm{~V})$. In general, the specific capacitance per unit area of all the electrodes analyzed in Table 1 and Figure 2(b) are higher than those reported by various authors in the field [38] [42] [43].

\subsection{Scanning Electron Microscopy}

Figure 3(a) presents the SEM micrograph of the PEDOT:PSS/PO ${ }_{4}$ coating by electrophoretic deposit. The micromorphology is densely porous and homogeneous. Amorphous reliefs appear as well as racemic clusters of polymer nanoparticle coalescence that give rise to supra structures with spheroidal shapes. The pores are nano-sized, the EDX mapping shows a uniform distribution of the elements on the surface, and the content of S, C and O are lower concerning the bulk polymer (Figure S1). Figure 3(b) shows the SEM micrograph of the EDOT-PY 0.9 - 0.1:PSS/ $/ \mathrm{PO}_{4}$ coating. A microporous and homogeneous surface is appreciated, no signs of delamination were seen, the copolymer produces porosities smaller in size than those observed in homopolymers, the surface area of the 
Table 1. Electrical conductivity, specific capacitance and charge storage capacity of different electrodes.

\begin{tabular}{cccc}
\hline Electrode & $\begin{array}{c}\text { Electrical } \\
\text { Conductivity } \\
\left(\mathrm{S}^{*} \mathrm{sq}\right)\end{array}$ & $\begin{array}{c}\text { Specific } \\
\text { Capacitance }\left(\mathrm{F} / \mathrm{cm}^{2}\right)\end{array}$ & $\begin{array}{c}\text { Charge Storage Capacity } \\
\left(\mathrm{mC} / \mathrm{cm}^{2}\right)\end{array}$ \\
\hline PEDOT PSS PO 4 & 0.015384615 & 1.2666666667 & 0.3546666667 \\
EDOT-PY 0.9 - 0.1 PSS PO & 0.081632653 & 2.023809524 & 0.5666666667 \\
Stainless steel & 8 & 0.091666667 & 0.0146666667 \\
PEDOT PSS & 0.33 & 2.854166667 & 0.685 \\
PPY PSS & $2.22222 \mathrm{E}-07$ & 2.267857143 & 0.635 \\
\hline
\end{tabular}

a)

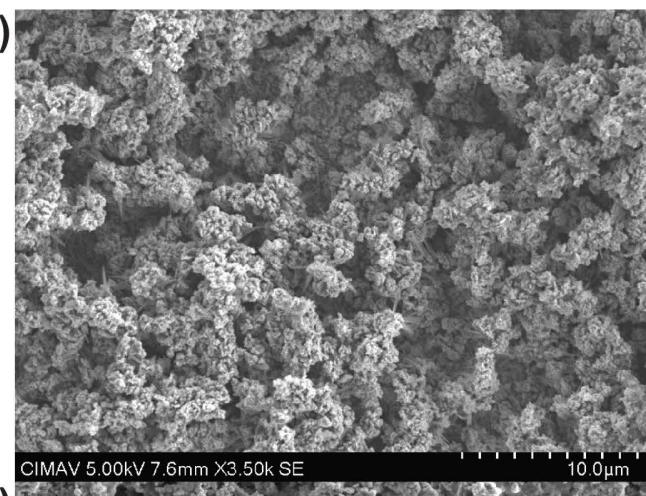

b)

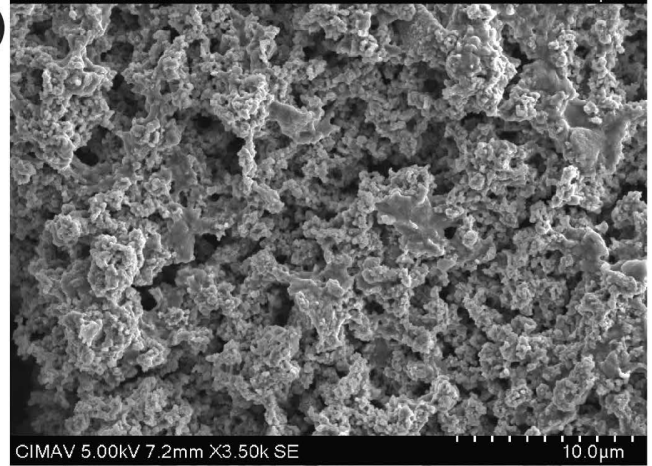

d)

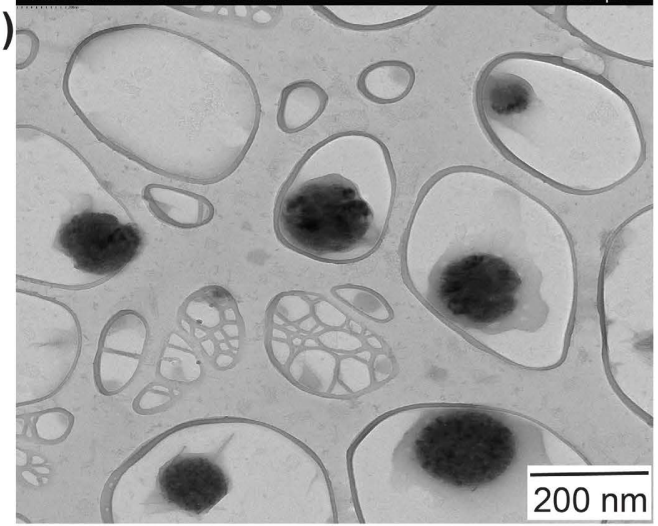

c)

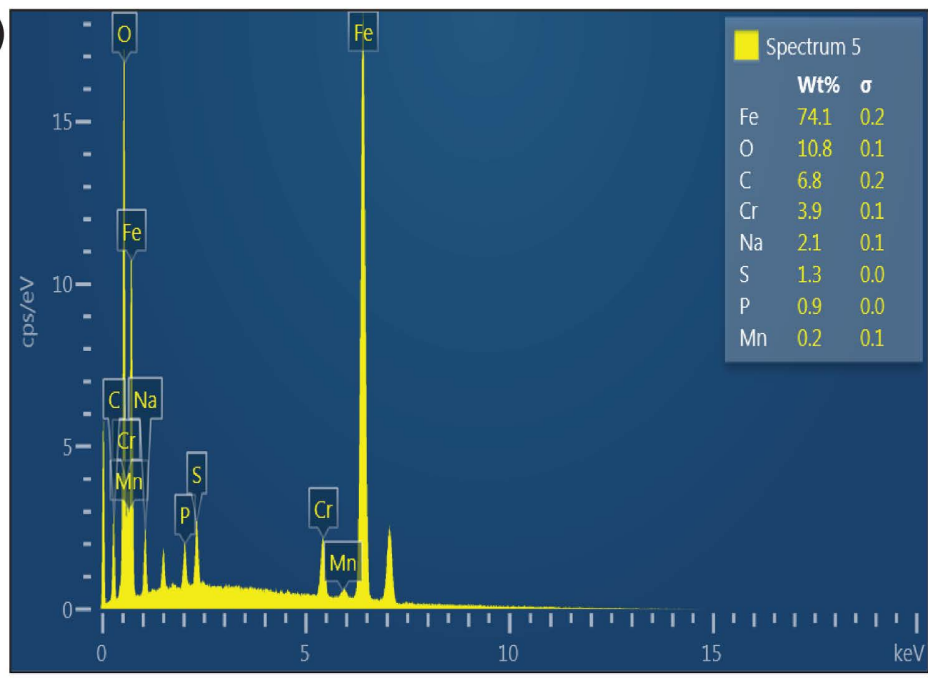

e)

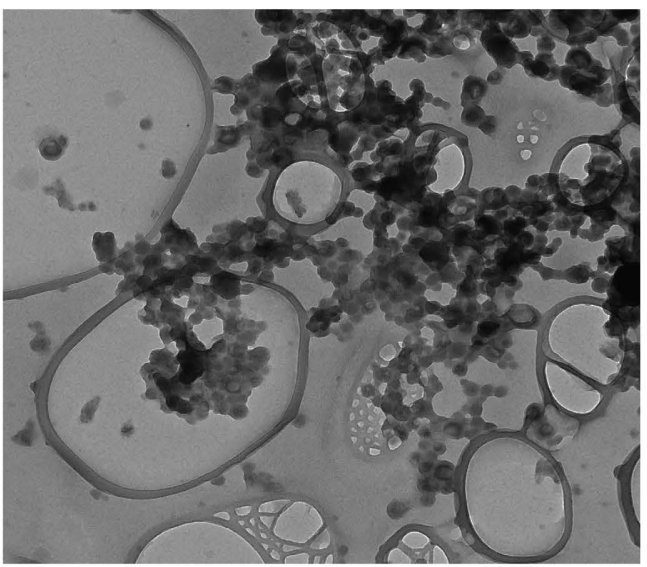

Figure 3. (a) SEM micrograph of stainless steel coated by EPD with PEDOT:PSS. (b) SEM micrograph of stainless steel coated by EPD with EDOT-PY. (c) EDX analysis of the coated surface. (d) TEM micrograph of $1 \mathrm{mg} / \mathrm{mL}$ suspension of PEDOT:PSS deposited on a lacey-carbon grid, detail of the nanoparticles formed. (e) TEM micrograph of $1 \mathrm{mg} / \mathrm{mL}$ suspension of EDOT-Py 0.9 0.1 :PSS deposited on a lacey-carbon grid. 
electrode increases drastically, the EDX mapping shows a uniform distribution of the elements on the surface, and the elemental ratio is similar to the one found for PEDOT:PSS $/ \mathrm{PO}_{4}$. However, there is an increase in sulfur content from $0.1 \%$ to $1.3 \%$, as well as a decrease in the content of $\mathrm{P}$ and $\mathrm{Na}$ (Figure 3(c)) concerning PEDOT, which indicates an increase in the number of polymer particles deposited on the surface and a decrease in phosphorous-sodium complexes associated with carry-over and coalescence on the electrode. The reason fundamentally may be due to the size of the copolymer particle that is smaller regarding PEDOT ( $\sim 30 \mathrm{~nm}$ vs. $200 \mathrm{~nm}$, see TEM characterization). In general, it is homogeneous, although micrometric grooves are observed which generate laminar segments along with the electrode, which could generate long-term delamination. In the cycles evaluated in this study, no loss of electrode integrity or variations in resistance and signal-to-noise ratio were observed (see Conductivity and SNR below), which indicate that it is only a morphological effect of the macro-relief and obeys the mobility kinetics.

\subsection{Transmission Electron Microscopy}

Figure 3(d) corresponds to the TEM micrograph obtained from the PEDOT:PSS; $500 \mathrm{~nm}$ spheroidal particles were observed. Figure 3(e) shows the TEM micrograph of the EDOT-PY:PSS $0.9-0.1$ copolymer. It is possible to observe a change in morphology and particle size relative to PEDOT:PSS, which had already been observed in previous studies of potential $\mathrm{Z}$ and DLS, where it is shown that the copolymer with a small molar fraction of pyrrole drastically modifies its colloidal properties. The effect is corroborated by the decrease in particle size and the racemic morphology observed of multiple nanoparticles between 10 and $20 \mathrm{~nm}$ that form micrometric clusters. In Figure 3(b), we can see morphological characteristics that could be related to the nanometric detail of Figure 3(e). The electrophoretic deposit drives the massive coalescence and agglomeration of cathodically transported nanoparticles which eventually becomes a highly porous coating.

\subsection{Electrocardiogram Assays}

Electrocardiogram recordings were performed using the methodology described in the experimental section, which consists of placing active electrodes on the right and left forearm and a ground electrode on the test subject's ankle. The electrodes available for the electrophysiological test were flat stainless-steel sheets $(1.5 \times 0.5 \times 0.01 \mathrm{~cm})$ covered by electrophoretic deposit with EDOT-PY 0.9 - 0.1: $\mathrm{PSS} / \mathrm{PO}_{4}$. This electrode presented a voltametric profile close to the ideal in an EDLC, approximately $8 \mathrm{mg} / \mathrm{cm}^{2}$ of deposited mass, high capacitance per unit area, and an acceptable resistance $(49 \mathrm{ohms} / \mathrm{cm}$ ) as discussed above.

Figure 4 (blue ECG plot) shows the electrocardiogram obtained with EDOT-PY $0.9-0.1: \mathrm{PSS} / \mathrm{PO}_{4}$ electrode. In this plot, it is possible to observe typical bioelectric signals of the myocardium. With adequate precision it has been 


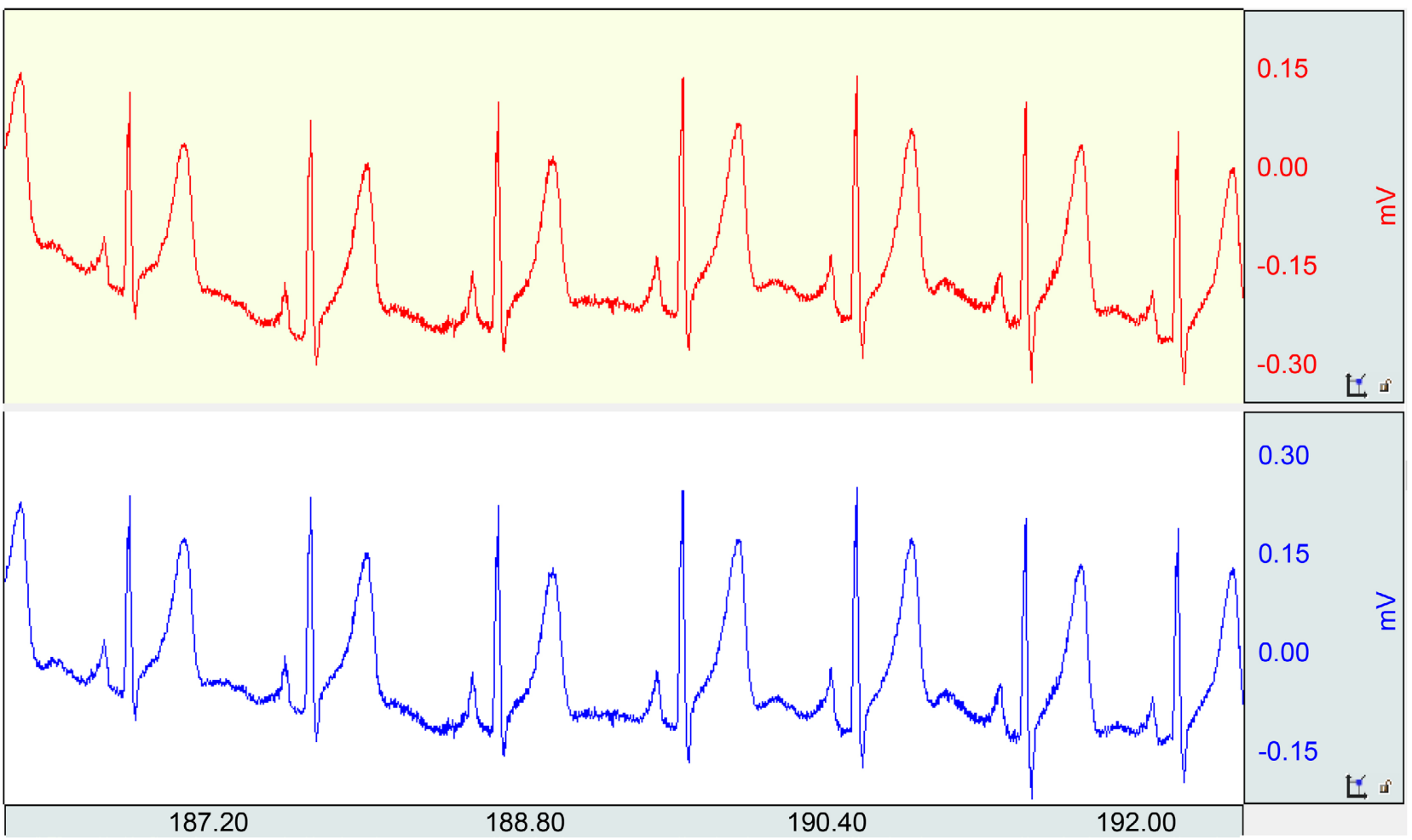

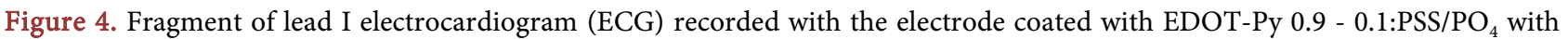
test subject at rest. ECG traces: Red trace corresponds to $\mathrm{Ag} / \mathrm{AgCl}$ biopac gel electrode; blue trace corresponds to EDOT-Py 0.9 $0.1: \mathrm{PSS} \mathrm{PO}_{4}$.

distinguished that [44]: the P wave corresponds to atrial depolarization, the QRS complex is associated with massive ventricular depolarization. The peak constitutes the highest signal intensity due to the massive contribution of ventricular fibers that reflect millions of action potentials of individual cardiomyocytes. After this event, the $\mathrm{T}$ wave is distinguished, which corresponds to ventricular repolarization. The frequencies between the $\mathrm{P}$ and $\mathrm{Q}$ waves are within $180-190$ $\mathrm{ms}$, while the S-T frequency is for less than $400 \mathrm{~ms}$, which indicates that there are no AV blocks. In general, the quality of the electrocardiogram is sufficiently satisfactory for diagnostic purposes [7]. The upper red plot of Figure 4 corresponds to the electrocardiogram recorded simultaneously with $\mathrm{Ag} / \mathrm{AgCl}$ Biopac contact electrodes. The recorded signals are less wide, the amplitude of the QRS complex wave is lower, and the sinusoidal shape of the $\mathrm{P}$ and $\mathrm{T}$ waves was observed. This is verified when performing the electronic analysis of the signals; both electrodes have a noise RMS close to $6 \mu \mathrm{V}$ (Figure 6(c)). However, the SNR of the EDOT-PY electrode is equal to $36 \mathrm{~dB}$ while the $\mathrm{Ag} / \mathrm{AgCl}$ electrode is close to $24 \mathrm{~dB}$ (Figure 6(b)).

This implies a better signal-to-noise ratio for the conductive copolymer electrode, which is strongly associated with surface conductivity and electrode capacitance. The copolymer coated electrode exhibits a surface conductivity of 0.08 $\mathrm{S}^{\star} \mathrm{sq}$ and capacitance of $2.02 \mathrm{~F} / \mathrm{cm}^{2}$ against a surface conductivity of $0.25 \mathrm{~S}^{*} \mathrm{sq}$ and a specific capacitance of $0.5 \mathrm{~F} / \mathrm{cm}^{2}$ for the $\mathrm{Ag} / \mathrm{AgCl}$ electrode. In a very gen- 
eral sense, it can be argued that CCBS copolymer electrodes have lower skin contact impedance than the commercial electrodes mostly used in commercial assays (Figure 6(a)). The results reflect differences in surface conductivity and capacitance per unit area. It has a significant effect on electrophysiological recordings and paves the way for future tests for stimulation and recording of electrical activity in neurons.

Figure 5 shows the ECG recordings obtained for the II, III and AVR leads. The same protocol described above was followed in this case. The only difference was the position of the electrodes on the test subject. The ECG segment shows typical signals for all recordings: P wave, QRS complex, and T90 wave. The recording is satisfactory for all leads. Not much thermal noise is observed (Figure 6(c)), and neither is the signal amplitude; for all of them is similar concerning the first lead signal amplitude. The electroactive area is the same for all electrodes and therefore the density of signals collected per unit area [45]. Any changes of the bioelectric signal amplitude are according to the electrical organ behavior and electrode positions, but not to the electrode sensitivity. Electrophysiological ECG tests revealed that the EDOT-PY 0.9 - 0.1:PSS/ $\mathrm{PO}_{4}$ electrode exhibits excellent properties for the collection of bioelectrical signals in humans, and of even higher quality than the commercial $\mathrm{Ag} / \mathrm{AgCl}$ electrodes. These results constitute a solid basis to continue the research and development of electrodes made with CCBS for electrophysiological and neurophysiological applications, as well as for the candidates to replace even the most expensive types of metallic electrodes that can be found in the market.

Figure 6(c) shows how thermal noise voltage varies depending on the electrode positions according to Einthoven's triangle. In the plot, it is possible to observe that the first, second, and third ECG leads exhibit similar skin impedance, but the AVR lead is lower than the other recordings. This implies that the position of the electrodes is highly dependent on the skin impedances obtained by CCBS electrodes as well as by $\mathrm{Ag} / \mathrm{AgCl}$ electrodes. However, the thermal noise voltage remains low for all ECG recordings.

We believe that both kinds of electrodes are sufficiently accurate for low noise electrocardiogram recording which would imply that the CCBS electrodes are as useful and effective as commercial Ag/AgCl ECG as discussed above.

Figure 6(d) represents the results related to the SNR electrodes after several times reused. Briefly, it consisted of placing three CCBS electrodes according to Einthoven's lead I and recording one ECG for two hours with normal subject test mobility throughout the assay. After that, the electrode was washed with water and ethanol several times and dried, then the same protocol was repeated two more times. We found that the SNR decreased in a monotonical way after each cycle. The electrode held $80 \%$ of the initial SNR value after being reused three times, however the SNR value was still high and acceptable for ECG recording with low noise. This implies that the CCBS electrodes probably work for long-term recordings or wearable applications. 


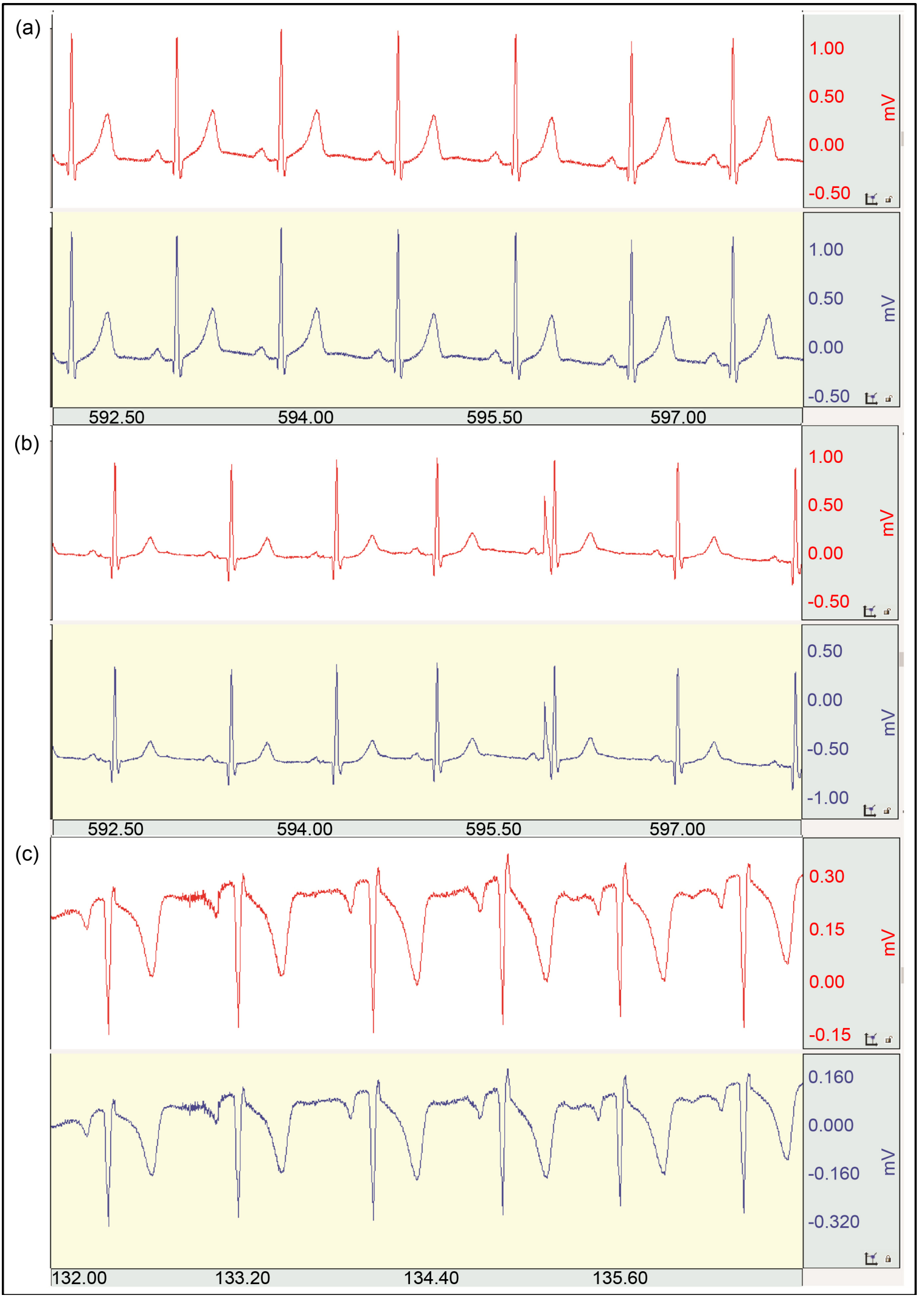

Figure 5. Fragment of electrocardiogram (ECG) recorded with the electrode coated with EDOT-PY 0.9 0.1:PSS/ $\mathrm{PO}_{4}$-P with test subject at rest. Inset normal ECG traces: (a) second lead, (b) third lead and, (c) AVR lead. Red trace corresponds to $\mathrm{Ag} / \mathrm{AgCl}$ biopac gel electrode, blue trace corresponds to EDOT-PY 0.9 - 0.1:PSS/PO . 

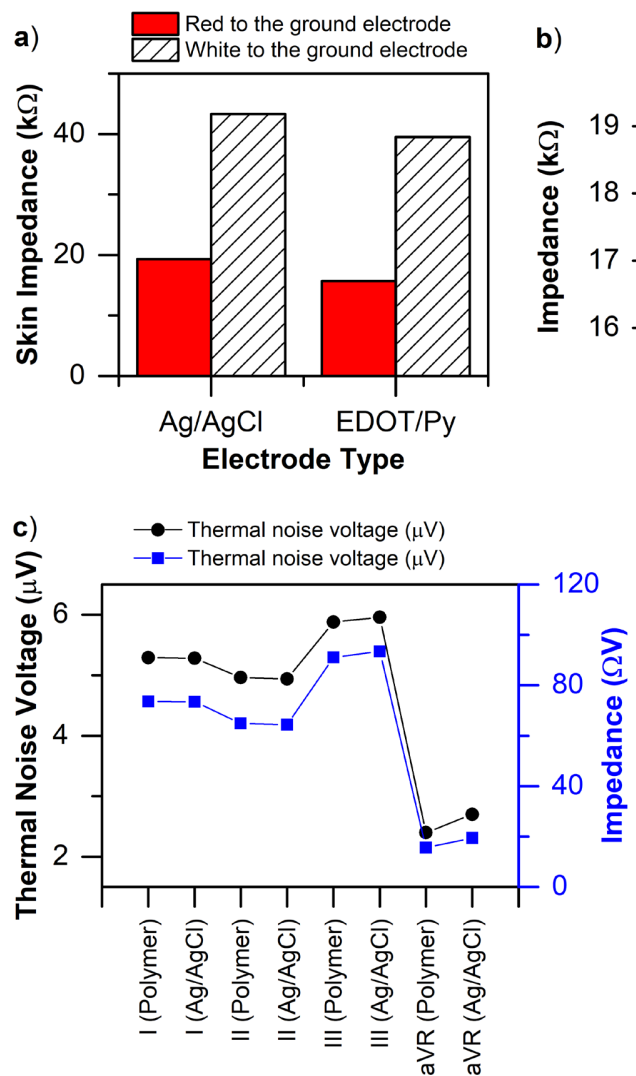

Derivation ECG

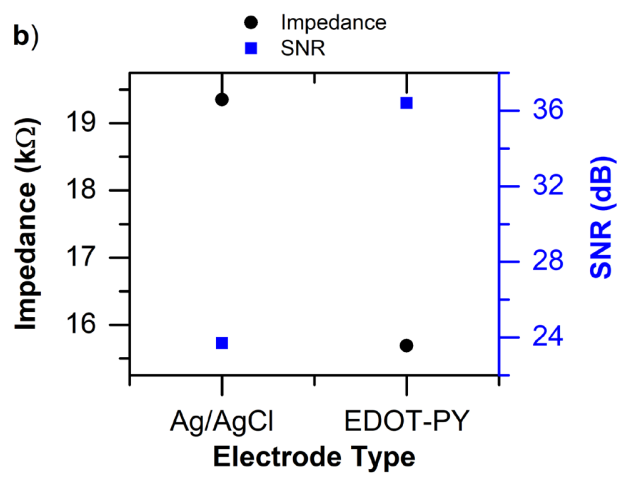

d)

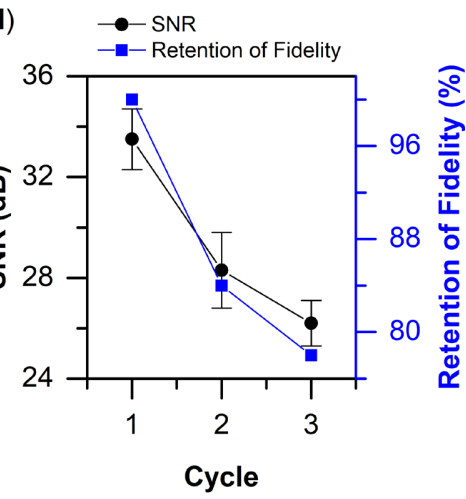

Figure 6. (a) Skin Impedance $\mathrm{Ag} / \mathrm{AgCl}$ commercial electrodes and EDOT-PY electrodes. The first column represents the skin impedance between red to the ground electrode; the second column represents the skin impedance between white to the ground electrode. (b) SNR analysis of the $\mathrm{Ag} / \mathrm{AgCl}$ electrode against electrodes coated with EDOT-PY 0.9 $0.1: \mathrm{PSS}_{\mathrm{PO}}$. (c) Thermal noise voltage and impedance ECG analysis for recordings obtained with EDOT-PY 0.9 - 0.1:PSS/ $\mathrm{PO}_{4}$ electrodes. (d) SNR retention after several reuse cycles.

\section{Conclusion}

The $\mathrm{Ag} / \mathrm{AgCl}$ electrodes exhibited a low specific capacitance per unit area (0.5 $\left.\mathrm{F} / \mathrm{cm}^{2}\right)$ as well as a narrow working potential window $(-0.045$ to $0.2 \mathrm{~V}$ vs. $\mathrm{Ag} / \mathrm{AgCl}$ ) compared with the CCBS electrodes manufactured in this work by electrophoretic deposition, which display high capacitances and a wide working potential window $(-0.7$ to $0.7 \mathrm{~V})$. It is possible to conclude that the EDOT:PY copolymer electrodes fabricated here possess high stability, which is one of the most desirable properties in long-term implants. Finally, electrophysiological tests showed high sensitivity to bioelectric signals when EDOT-PY $0.9-0.1 / \mathrm{PO}_{4}$ copolymer electrodes were used, which exhibit low thermal noise $(6 \mu \mathrm{V})$ and a high SNR (36 - $24 \mathrm{~dB})$. This allowed us to obtain defined electrocardiographic traces. The conductive copolymer material synthesized here is ideal for the medical diagnosis of cardiac disorders or musculoskeletal diseases. This work constitutes the first report of conductive copolymer electrodes manufactured by electrophoretic deposition successfully applied to electrophysiology recordings. 


\section{Acknowledgements}

This work was supported by a CONACYT-Mexico PhD Fellowship given to Manuel Eduardo Martínez-Cartagena, Project FONCYT COAH-2020-C14-B007: Biomimetic synthesis of bioinspired semiconductor conjugated copolymers as neuronal interfaces and BASIC SCIENCE: Project CB2016-287954-Q. Hematin supported on nanostructured carbon nitride layers: Biomimetic catalyst for the synthesis of polyaniline. Thanks to Professor Felipe Rizo, Dean of Engineering Science Center and Ing. Carlos Edgar López-Barrera from Universidad Autónoma de Aguascalientes, México, for the technical and academic support. Thanks to Laura Elias for her English revision and edition. Thanks to Dr. Nicolas Muzzio and Professor Gabriela Romero from UTSA for the technical and academic support.

\section{Conflicts of Interest}

The authors declare no conflicts of interest regarding the publication of this paper.

\section{References}

[1] Kolanowska, A., Herman, A.P., Jȩdrysiak, R.G. and Boncel, S. (2021) Carbon Nanotube Materials for Electrocardiography. RSC Advances, 11, 3020-3042.

https://doi.org/10.1039/D0RA08679G

[2] Bernal-Martínez, J., Seseña-Rubfiaro, A., Godínez-Fernández, R. and Aguilar-Elguezabal, A. (2016) Electrodes Made of Multi-Wall Carbon Nanotubes on PVDF-Filters Have Low Electrical Resistance and Are Able to Record Electrocardiograms in Humans. Microelectron Engineering, 166, 10-14.

https://doi.org/10.1016/j.mee.2016.09.006

[3] Wang, L., Zhang, F., Lu, K., et al. (2020) Nano-Copper Enhanced Flexible Device for Simultaneous Measurement of Human Respiratory and Electro-Cardiac Activities. Journal of Nanobiotechnology, 18, 82. https://doi.org/10.1186/s12951-019-0562-3

[4] Ullas Pradhan, U., Reddy, N., Chandrashekar, K. and Mohan, C.B. (2021) Titanium Dioxide Based Bioelectric Sensor for the Acquisition of Electrocardiogram Signals. Microchemical Journal, 160, Article ID: 105656. https://doi.org/10.1016/j.microc.2020.105656

[5] Ren, X., Yang, M., Yang, T., et al. (2021) Highly Conductive PPy-PEDOT:PSS Hybrid Hydrogel with Superior Biocompatibility for Bioelectronics Application. ACS Applied Materials \& Interfaces, 13, 25374-25382. https://doi.org/10.1021/acsami.1c04432

[6] Nigusse, A.B., Mengistie, D.A., Malengier, B., Tseghai, G.B. and Van Langenhove, L. (2021) Wearable Smart Textiles for Long-Term Electrocardiography Monitoring-A Review. Sensors, 21, 4174. https://doi.org/10.3390/s21124174

[7] Bihar, E., Roberts, T., Saadaoui, M., et al. (2017) Inkjet-Printed PEDOT:PSS Electrodes on Paper for Electrocardiography. Advanced Healthcare Materials, 6, Article ID: 1601167. https://doi.org/10.1002/adhm.201601167

[8] Rossetti, N., Luthra, P., Hagler, J., et al. (2019) Poly(3,4-ethylenedioxythiophene) (PEDOT) Coatings for High-Quality Electromyography Recording. ACS Applied 
Bio Materials, 2, 5154-5163. https://doi.org/10.1021/acsabm.9b00809

[9] Zahed, M.A., Das, P.S., Maharajan, P., et al. (2020) Flexible and Robust Dry Electrodes Based on Electroconductive Polymer Spray-Coated 3D Porous Graphene for Long-Term Electrocardiogram Signal Monitoring System. Carbon, 165, 26-36. https://doi.org/10.1016/j.carbon.2020.04.031

[10] Sinha, S.K., Noh, Y., Reljin, N., et al. (2017) Screen-Printed PEDOT:PSS Electrodes on Commercial Finished Textiles for Electrocardiography. ACS Applied Materials \& Interfaces, 9, 37524-37528. https://doi.org/10.1021/acsami.7b09954

[11] Mihara, S., Lee, H.L. and Takeoka, S. (2020) Electrocardiogram Measurements in Water Using Poly(3,4-ethylene dioxythiophene):Poly(styrene sulfonate) Nanosheets Waterproofed by Polyurethane Film. MRS Communications, 10, 628-635. https://doi.org/10.1557/mrc.2020.72

[12] Lee, Y., Yim, S.-G., Lee, G.W., et al. (2020) Self-Adherent Biodegradable GelatinBased Hydrogel Electrodes for Electrocardiography Monitoring. Sensors, 20, 5737. https://doi.org/10.3390/s20020573

[13] Trindade, I.G., Martins, F. and Baptista, P. (2015) High Electrical Conductance Poly(3,4-ethylenedioxythiophene) Coatings on Textile for Electrocardiogram Monitoring. Synthetic Metals, 210, 179-185. https://doi.org/10.1016/j.synthmet.2015.09.024

[14] Romero-García, J., Ledezma-Pérez, A., Martínez-Cartagena, M., et al. (2019) Radical Addition Polymerization: Enzymatic Template-Free Synthesis of Conjugated Polymers and Their Nanostructure Fabrication. In: Bruns, N. and Loos, K., Eds., Methods in Enzymology, Volume 627: Enzymatic Polymerization, Academic Press, Inc., Amsterdam, 321-337. https://doi.org/10.1016/bs.mie.2019.08.009

[15] Tierrablanca, E., Romero-García, J., Roman, P. and Cruz-Silva, R. (2010) Biomimetic Polymerization of Aniline Using Hematin Supported on Halloysite Nanotubes. Applied Catalysis A: General, 381, 267-273.

https://doi.org/10.1016/j.apcata.2010.04.021

[16] Valtakari, D., Liu, J., Kumar, V., Xu, C., Toivakka, M. and Saarinen, J. (2015) Conductivity of PEDOT:PSS on Spin-Coated and Drop Cast Nanofibrillar Cellulose Thin Films. Nanoscale Research Letters, 10, 1-10. https://doi.org/10.1186/s11671-015-1093-y

[17] Benoudjit, A., Bader, M.M. and Wan Salim, W.W.A. (2018) Study of Electropolymerized PEDOT:PSS Transducers for Application as Electrochemical Sensors in Aqueous Media. Sensing and Bio-Sensing Research, 17, 18-24. https://doi.org/10.1016/j.sbsr.2018.01.001

[18] Garma, L.D., Ferrari, L.M., Scognamiglio, P., Greco, F. and Santoro, F. (2019) Inkjet-Printed PEDOT:PSS Multi-Electrode Arrays for Low-Cost in Vitro Electrophysiology. Lab on a Chip, 19, 3776-3786. https://doi.org/10.1039/C9LC00636B

[19] Yuk, H., Lu, B., Lin, S., et al. (2020) 3D Printing of Conducting Polymers. Nature Communications, 11, 1-8. https://doi.org/10.1038/s41467-020-15316-7

[20] Wang, F., Zhang, X., Ma, Y. and Yang, W. (2018) Synthesis of HNTs@PEDOT Composites via in Situ Chemical Oxidative Polymerization and Their Application in Electrode Materials. Applied Surface Science, 427, 1038-1045. https://doi.org/10.1016/j.apsusc.2017.08.155

[21] Ouyang, S., Xie, Y., Wang, D., et al. (2015) Surface Patterning of PEDOT:PSS by Photolithography for Organic Electronic Devices. Journal of Nanomaterials, 2015, 4. https://doi.org/10.1155/2015/603148

[22] Lattach, Y., Deniset-Besseau, A., Guigner, J.M. and Remita, S. (2013) Radiation Che- 
mistry as an Alternative Way for the Synthesis of PEDOT Conducting Polymers Under "Soft" Conditions. Radiation Physics and Chemistry, 82, 44-53. https://doi.org/10.1016/j.radphyschem.2012.09.009

[23] Sandoval, A.P., Suárez-Herrera, M.F. and Feliu, J.M. (2015) IR and Electrochemical Synthesis and Characterization of Thin Films of PEDOT Grown on Platinum Single Crystal Electrodes in [EMMIM] $\mathrm{Tf}_{2} \mathrm{~N}$ Ionic Liquid. Beilstein Journal of Organic Chemistry, 11, 348-357. https://doi.org/10.3762/bjoc.11.40

[24] Bruno, F.F., Fossey, S.A., Nagarajan, S., et al. (2006) Biomimetic Synthesis of Water-Soluble Conducting Copolymers/Homopolymers of Pyrrole and 3,4-Ethylenedioxythiophene. Biomacromolecules, 7, 586-589.

https://doi.org/10.1021/bm0506501

[25] Luna-Zaragoza, D., Romero-Guzmán, E.T. and Reyes-Gutiérrez, L.R. (2009) Surface and Physicochemical Characterization of Phosphates Vivianite, $\mathrm{Fe}_{2}\left(\mathrm{PO}_{4}\right)_{3}$ and Hydroxyapatite, $\mathrm{Ca}_{5}\left(\mathrm{PO}_{4}\right)_{3} \mathrm{OH}$. Journal of Minerals \& Materials Characterization \& Engineering, 8, 591-609. https://doi.org/10.4236/jmmce.2009.88052

[26] Wang, X., Kyaw, A.K.K., Yin, C., et al. (2018) Enhancement of Thermoelectric Performance of PEDOT:PSS Films by Post-Treatment with a Superacid. RSC Advances, 8, 18334-18340. https://doi.org/10.1039/C8RA02058B

[27] Zhou, J., Li, E.Q., Li, R., et al. (2015) Semi-Metallic, Strong and Stretchable WetSpun Conjugated Polymer Microfibers. Journal of Materials Chemistry C, 3, 25282538. https://doi.org/10.1039/C4TC02354D

[28] Bahry, T., Cui, Z., Deniset-Besseau, A., et al. (2018) An Alternative Radiolytic Route for Synthesizing Conducting Polymers in an Organic Solvent. New Journal of Chemistry, 42, 8704-8716. https://doi.org/10.1039/C8NJ01041B

[29] Kim, J., Park, C., Im, S., Lee, H. and Kim, J.H. (2019) Effect of Molecular Weight Distribution of PSSA on Electrical Conductivity of PEDOT:PSS. RSC Advances, 9, 4028-4034. https://doi.org/10.1039/C8RA09919G

[30] Anothumakkool, B., Soni, R., Bhange, S.N. and Kurungot, S. (2015) Novel Scalable Synthesis of Highly Conducting and Robust PEDOT Paper for a High Performance Flexible Solid Supercapacitor. Energy and Environmental Science, 8, 1339-1347. https://doi.org/10.1039/C5EE00142K

[31] Wang, X., Ge, M. and Feng, G. (2015) The Effects of DMSO on Structure and Properties of PVA/PEDOT:PSS Blended Fiber. Fibers and Polymers, 16, 2578-2585. https://doi.org/10.1007/s12221-015-5616-Z

[32] Roy, S., Fortier, J.M., Nagarajan, R., et al. (2002) Biomimetic Synthesis of a Water Soluble Conducting Molecular Complex of Polyaniline and Lignosulfonate. Biomacromolecules, 3, 937-941. https://doi.org/10.1021/bm0255138

[33] Zhang, K., Zhang, L.L., Zhao, X.S. and Wu, J. (2010) Graphene/Polyaniline Nanofiber Composites as Supercapacitor Electrodes. Chemistry of Materials, 22, 1392-1401. https://doi.org/10.1021/cm902876u

[34] Mostafaei, A. and Zolriasatein, A. (2012) Synthesis and Characterization of Conducting Polyaniline Nanocomposites Containing ZnO Nanorods. Progress in Natural Science: Materials International, 22, 273-280.

https://doi.org/10.1016/j.pnsc.2012.07.002

[35] Damodaran, R. and Moudgil, B.M. (1993) Electrophoretic Deposition of Calcium Phosphates from Non-Aqueous Media. Colloids and Surfaces A: Physicochemical and Engineering Aspects, 80, 191-195.

https://doi.org/10.1016/0927-7757(93)80198-N 
[36] Kalinina, E.G. and Pikalova, E.Y. (2019) New Trends in the Development of Electrophoretic Deposition Method in the Solid Oxide Fuel Cell Technology: Theoretical Approaches, Experimental Solutions and Development Prospects. Russian Chemical Reviews, 88, 1179-1219. https://doi.org/10.1070/RCR4889

[37] Martinez-Cartagena, M.E. (2017) Compósitos Polianilina-Nitruro de Carbono Polimérico Modificado Orientados al Almacenamiento de Energía. Centro de Investigacion en Quimica Aplicada.

[38] Liu, Y., Shi, K. and Zhitomirsky, I. (2015) Azopolymer Triggered Electrophoretic Deposition of $\mathrm{MnO}_{2}$-Carbon Nanotube Composites and Polypyrrole Coated Carbon Nanotubes for Supercapacitors. Journal of Materials Chemistry A, 3, 16486-16494. https://doi.org/10.1039/C5TA03969J

[39] Wu, W., Wang, J., Zhang, T., et al. (2019) Controllable Synthesis of Ag/AgCl@MIL88A via in Situ Growth Method for Morphology-Dependent Photocatalytic Performance. Journal of Materials Chemistry C, 7, 5451-5460.

https://doi.org/10.1039/C9TC00398C

[40] Wu, S.Y., Hou, H.S., Sun, Y.S., Cheng, J.Y. and Lo, K.Y. (2015) Correlation between Cell Migration and Reactive Oxygen Species under Electric Field Stimulation. Biomicrofluidics, 9, 054120. https://doi.org/10.1063/1.4932662

[41] Peng, J., Deng, Y., Wang, D., Jin, X. and Chen, G.Z. (2009) Cyclic Voltammetry of Electroactive and Insulative Compounds in Solid State: A Revisit of $\mathrm{AgCl}$ in Aqueous Solutions Assisted by Metallic Cavity Electrode and Chemically Modified Electrode. Journal of Electroanalytical Chemistry, 627, 28-40. https://doi.org/10.1016/j.jelechem.2008.12.016

[42] Lota, G., Fic, K. and Frackowiak, E. (2011) Carbon Nanotubes and Their Composites in Electrochemical Applications. Energy \& Environmental Science, 4, 1592. https://doi.org/10.1039/c0ee00470g

[43] Hassan, S., Suzuki, M. and El-Moneim, A.A. (2014) Facile Synthesis of $\mathrm{MnO}_{2}$ / Graphene Electrode by Two-Steps Electrodeposition for Energy Storage Application. International Journal of Electrochemical Science, 9, 8340-8354. http://electrochemsci.org/papers/vol9/91208340.pdf

[44] Ankhili, A., Tao, X., Cochrane, C., et al. (2018) Comparative Study on Conductive Knitted Fabric Electrodes for Long-Term Electrocardiography Monitoring: Silver-Plated and PEDOT:PSS Coated Fabrics. Sensors, 18, 3890. https://doi.org/10.3390/s18113890

[45] Nyberg, T., Shimada, A. and Torimitsu, K. (2007) Ion Conducting Polymer Microelectrodes for Interfacing with Neural Networks. Journal of Neuroscience Methods, 160, 16-25. https://doi.org/10.1016/j.jneumeth.2006.08.008 


\section{Supporting Information}

Table S1. Summarizing of Several IR Vibrational modes on PEDOT:PSS, PPY:PSS and EDOT-Py:PSS copolymers at different molar fractions.

\begin{tabular}{|c|c|c|c|c|c|c|c|c|}
\hline & & & & $\begin{array}{l}\text { EDOT-Py } \\
\text { PSS }(2: 1)\end{array}$ & & $\begin{array}{l}\text { EDOT-Py } \\
\text { PSS }(2: 1) \\
(1: 40 \\
\text { PSS/TSA) }\end{array}$ & & $\begin{array}{c}\text { EDOT-Py } \\
\text { PSS-PS (2:1) } \\
(1: 40 \\
\text { PSS-PS/TSA) }\end{array}$ \\
\hline Asignation & PEDOT PSS & PPY PSS & $0.9-0.1$ & $0.7-0.3$ & $0.9-0.1$ & $0.7-0.3$ & $0.9-0.1$ & $0.7-0.3$ \\
\hline$v(\mathrm{C}=\mathrm{O})$ & & & & 1726 & & & & \\
\hline$v(\mathrm{C}=\mathrm{N})$ & & 1709 & & & & & & \\
\hline $\begin{array}{c}v(\mathrm{C}=\mathrm{N}), \delta(\mathrm{NH}) \\
v(-\mathrm{OH} \text { agua })\end{array}$ & 1631 & 1626 & 1637 & 1637 & & & & \\
\hline $\begin{array}{c}v(\mathrm{C}=\mathrm{C}) \text { asym, } \\
\text { heterocyclic ring } \\
\text { quinoide ( } \mathrm{p} \text { doped) }\end{array}$ & 1599 & 1600 & & & & & & \\
\hline $\begin{array}{l}\qquad(\mathrm{C}=\mathrm{C}) \\
\text { asymbenzenoide } \\
\text { heterocycle }\end{array}$ & 1545 & & & & 1511 & & 1510 & 1510 \\
\hline $\begin{array}{c}v(\mathrm{C}=\mathrm{C}) \text { sym, } \\
\text { benzenoide } \\
\text { heterocycle } \\
\text { ( } \mathrm{n} \text { doped) }\end{array}$ & 1493 & 1493 & 1484 & 1484 & 1479 & 1480 & 1479 & 1479 \\
\hline $\begin{array}{c}v(\mathrm{C}=\mathrm{C}) \text { sym, } \\
\text { heterocyclic ring } \\
\text { quinoide ( } \mathrm{p} \text { doped) }\end{array}$ & 1477 & 1443 & 1444 & 1444 & & & 1443 & 1443 \\
\hline $\begin{array}{l}v(\mathrm{C}-\mathrm{C}) \text { asym } \\
\text { heterocycle }\end{array}$ & 1445 & & & & & & & \\
\hline$v\left(\mathrm{SO}_{3}\right)$ asym TSA & 1409 & 1407 & 1393 & 1393 & 1390 & & 1392 & 1392 \\
\hline $\begin{array}{c}v(\mathrm{C}-\mathrm{C}) \text { sym, } \\
v(\mathrm{C}-\mathrm{O}-\mathrm{C}) \text { EDOT } \\
\text { Oxyethylene ring }\end{array}$ & 1352 & & 1362 & 1362 & & 1357 & & \\
\hline$\delta(\mathrm{C}-\mathrm{N}), v(\mathrm{~N}-\mathrm{H})$ & & 1300 & & & 1306 & & 1307 & 1307 \\
\hline$\delta(\mathrm{C}-\mathrm{C})$ on plane ring & 1209 & 1209 & 1221 & 1221 & & & & \\
\hline$v\left(\mathrm{SO}_{3}\right)$ & 1172 & 1172 & 1178 & 1178 & 1178 & 1178 & 1180 & 1180 \\
\hline $\begin{array}{l}\delta(\mathrm{C}-\mathrm{O}-\mathrm{C}) \\
\delta(\mathrm{C}-\mathrm{H}) \text { on } \\
\text { plane ring }\end{array}$ & 1126 & 1126 & 1131 & 1131 & 1133 & 1133 & 1140 & 1140 \\
\hline$\delta(\mathrm{S}-\mathrm{O}-\mathrm{R}) \mathrm{TSA}$ & 1077 & 1077 & 1083 & 1083 & 1081 & 1081 & 1084 & 1084 \\
\hline $\begin{array}{c}\delta(\mathrm{C}-\mathrm{C}) \\
\text { heterocyclic ring }\end{array}$ & 1033 & 1033 & 1038 & 1038 & 1050 & 1050 & 1050 & 1050 \\
\hline $\begin{array}{c}\delta(\mathrm{C}-\mathrm{H}) \\
\text { outplane ring }\end{array}$ & 1006 & 1006 & 1011 & 1011 & 1007 & 1007 & 1007 & 1007 \\
\hline$\delta(\mathrm{C}-\mathrm{H})$ wagging & 976 & 948 & 992 & 992 & 967 & 967 & 974 & 974 \\
\hline $\begin{array}{c}\delta(\mathrm{C}-\mathrm{S}) \\
\text { thiophenic ring }\end{array}$ & 923 & & 941 & 941 & & & & \\
\hline $\begin{array}{c}\delta(\mathrm{C}-\mathrm{C}) \\
\text { heterocyclic ring }\end{array}$ & 832 & 835 & 841 & 841 & & & & \\
\hline
\end{tabular}




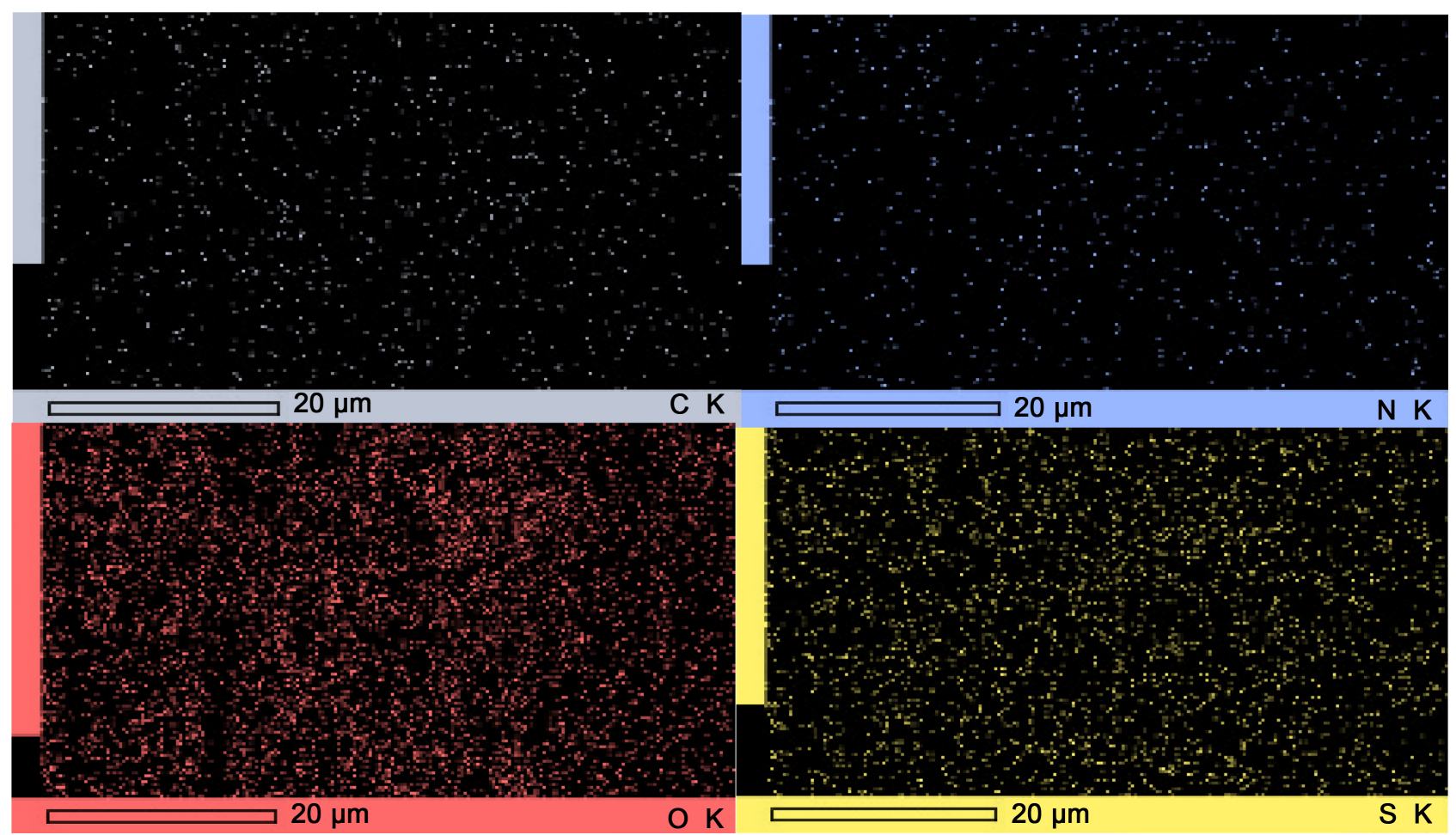

Figure S1. EDX element mapping results of PEDOT:PSS/PO $\mathrm{P}_{4}$ electrophorectic deposit on stainless steel. 